\title{
Development and Validation of the Cluster Headache Screening Questionnaire
}

\author{
Pil-Wook Chung ${ }^{a}$, Soo-Jin Cho ${ }^{b}$ \\ Byung-Kun Kim ${ }^{\circ}$ \\ Soo-Kyoung Kim ${ }^{\mathrm{d}}$ \\ Mi Ji Lee ${ }^{e}$, Yun-Ju Choi ${ }^{f}$ \\ Jeong Wook Park ${ }^{g}$, \\ Byung-Su Kim ${ }^{h}$, Kyungmi Ohi \\ Heui-Soo Moon ${ }^{a}$, Tae-Jin Song ${ }^{j}$ \\ Danbee Kang ${ }^{k, l}$, Juhee Cho ${ }^{k, l}$ \\ Chin-Sang Chung ${ }^{\mathrm{e}}$ \\ ${ }^{a}$ Department of Neurology, Kangbuk \\ Samsung Hospital, Sungkyunkwan \\ University School of Medicine, Seoul, \\ Korea \\ ${ }^{b}$ Department of Neurology, Dongtan \\ Sacred Heart Hospital, Hallym University \\ College of Medicine, Hwaseong, Korea \\ 'Department of Neurology, Eulji Hospital, \\ Eulji University, Seoul, Korea \\ dDepartment of Neurology, Gyeonsang \\ National University College of Medicine, \\ Jinju, Korea \\ eDepartment of Neurology, Neuroscience \\ Center, Samsung Medical Center, \\ Sungkyunkwan University School of \\ Medicine, Seoul, Korea \\ fDepartment of Neurology, Presbyterian \\ Medical Center, Jeonju, Korea \\ ${ }^{9}$ Department of Neurology, Uijeongbu St. \\ Mary's Hospital, The Catholic University \\ of Korea, Uijeongbu, Korea \\ hDepartment of Neurology, Bundang \\ Jesaeng General Hospital, Daejin \\ Medical Center, Seongnam, Korea \\ 'Department of Neurology, Korea \\ University College of Medicine, Seoul, \\ Korea \\ 'Department of Neurology, Ewha \\ Womans University College of Medicine, \\ Seoul, Korea \\ ${ }^{k}$ Department of Clinical Research Design \\ and Evaluation, SAIHST, Sungkyunkwan \\ University, Seoul, Korea \\ 'Center for Clinical Epidemiology, \\ Samsung Medical Center, \\ Sungkyunkwan University School of \\ Medicine, Seoul, Korea
}

Received August 13, 2018

Revised September 17, 2018

Accepted September 19, 2018

\section{Correspondence}

Soo-Jin Cho, $\mathrm{MD}, \mathrm{PhD}$

Department of Neurology,

Dongtan Sacred Heart Hospital,

Hallym University College of Medicine,

7 Keunjaebong-gil,

Hwaseong 18450, Korea

Tel +82-31-8086-2310

Fax +82-31-8086-2317

E-mail dowonc@naver.com
Background and Purpose Cluster headache $(\mathrm{CH})$ is frequently either not diagnosed or the diagnosis is delayed. We addressed this issue by developing the self-administered Cluster Headache Screening Questionnaire (CHSQ).

Methods Experts selected items from the diagnostic criteria of $\mathrm{CH}$ and the characteristics of migraine. The questionnaire was administered to first-visit headache patients at nine headache clinics. The finally developed CHSQ included items based on the differences in responses between $\mathrm{CH}$ and non- $\mathrm{CH}$ patients, and the accuracy and reliability of the scoring model were assessed.

Results Forty-two patients with $\mathrm{CH}, 207$ migraineurs, 73 with tension-type headache, and 18 with primary stabbing headache were enrolled. The CHSQ item were scored as follows: 3 points for ipsilateral eye symptoms, agitation, and duration; 2 points for clustering patterns; and 1 point for the male sex, unilateral pain, disability, ipsilateral nasal symptoms, and frequency. The total score of the CHSQ ranged from 0 to 16. The mean score was higher in patients with $\mathrm{CH}$ than in non- $\mathrm{CH}$ patients $(12.9$ vs. $3.4, p<0.001)$. At a cutoff score of $>8$ points, the CHSQ had a sensitivity, specificity, positive predictive value, and negative predictive value of $95.2 \%, 96 \%$, $76.9 \%$, and $99.3 \%$, respectively.

Conclusions The CHSQ is a reliable screening tool for the rapid identification of $\mathrm{CH}$.

Key Words cluster headache, diagnosis, migraine, prevalence, questionnaire, screening.

\section{INTRODUCTION}

Cluster headache $(\mathrm{CH})$ is characterized by severe recurrent unilateral head pain, ipsilateral cranial autonomic symptoms, and an impaired quality of life. ${ }^{1-3}$ Although little is known about the population-based epidemiology of $\mathrm{CH}$, a meta-analysis found wide ranges for the lifetime and 1-year prevalence rates, of 56-381 per 100,000 and 3-150 per 100,000, respectively. ${ }^{4}$ Men are reportedly 4.3-7 times more likely to be affected than women, with a peak age range at onset of 20-31 years. ${ }^{5,6}$ Furthermore, $\mathrm{CH}$ has a major socioeconomic impact in general due to both the direct healthcare costs and the indirect costs caused by loss of working capacity during the active working period. ${ }^{7}$

Despite $\mathrm{CH}$ being a very severe type of headache with distinct clinical features, the condition is underdiagnosed and undertreated. Several studies have found that diagnosis delays longer than 3 years are common in patients with $\mathrm{CH}^{8-10}$ which may be due to this severe unilateral type of headache being misdiagnosed as migraine. The relatively low prevalence, low public awareness, and small number of medical personnel specialized in primary headache disorders further contribute to the diagnosis delay of $\mathrm{CH}$. However, $\mathrm{CH}$ is not uncommon in headache clinics, and the established treatments for $\mathrm{CH}$ such as triptans, oxygen

( $)$ This is an Open Access article distributed under the terms of the Creative Commons Attribution Non-Commercial License (https://creativecommons.org/licenses/by-nc/4.0) which permits unrestricted non-commercial use, distribution, and reproduction in any medium, provided the original work is properly cited. 
therapy, and occipital steroid injection demand early diagnosis and rapid therapeutic approaches. ${ }^{11,12}$

Several self-administered screening questionnaires for identifying $\mathrm{CH}$ have been developed with the aim of expediting the diagnosis of $\mathrm{CH}^{13-15}$ Although the previously reported $\mathrm{CH}$ screening tools exhibited good validity and reliability, they have some limitations. Previous studies have validated these self-administered questionnaires in mixed cohorts of first-visit or previously diagnosed patients with $\mathrm{CH}$ or selfreported $\mathrm{CH}$ patients. ${ }^{13,14}$ The inclusion of patients diagnosed with $\mathrm{CH}$ or self-reported $\mathrm{CH}$ may result in bias in the questionnaire responses; namely, patients who are aware of $\mathrm{CH}$ may provide more-positive responses to questions related to the characteristic symptoms of $\mathrm{CH}$. Previous studies calculated the positive predictive value (PPV) and negative predictive value (NPV) based on comparisons of small numbers of patients with other headache disorders. Considering the relatively low prevalence of $\mathrm{CH}$ compared with migraine, screening tools specific for $\mathrm{CH}$ need to be developed and validated in a cohort of sufficient size with common headache disorders, for application in real-world situations such as a headache clinic or population studies. ${ }^{16}$

The objective of this study was to develop a simple and reliable screening tool to identify patients experiencing $\mathrm{CH}$ from among first-visit patients with a headache.

\section{METHODS}

\section{Participants}

The study participants were recruited from March to August 2017 at neurology clinics across nine hospitals in Korea. Subjects were eligible if they were first-visit headache patients, before completing the questionnaire, and were aged 19-65 years. Headache specialists examined the patients after they had answered the self-administered questionnaire. Each of the participating investigators made diagnoses according to the criteria of the International Classification of Headache Disorder, Third Edition, beta version (ICHD-3 $\beta$ ). ${ }^{17}$ We included patients diagnosed with $\mathrm{CH}$ and probable $\mathrm{CH}$. We additionally included patients who met the criteria of a definite diagnosis of migraine, tension-type headache (TTH), or primary stabbing headache (PSH), since the purpose of this study was to develop a simple screening tool for identifying $\mathrm{CH}$ from among first-visit primary headache patients. Patients with cognitive or psychologic impairment, secondary headache disorder, and other types of headache disorder were excluded. The goal was to recruit 300 patients, based on the minimum number of participants required for tool validation test statistics. ${ }^{18}$ Considering the low prevalence of $\mathrm{CH}$, we planned to recruit at least 30 patients with $\mathrm{CH}$ so that they comprised $\geq 10 \%$ of the study population. The final study population comprised 340 patients; 42 patients with $\mathrm{CH}$ and 298 patients with non- $\mathrm{CH}$. All of the study procedures were approved by the institutional review boards at each participating hospital, and the institutional review board allowed the requirement for informed consent to be waived considering that the questionnaire was administered prior to the diagnosis and there was no risk of infringing personal information (2016-396-I, KBSMC 2016-10-031).

\section{Questionnaire development}

To develop a tool for screening $\mathrm{CH}$ at the outpatient clinic, we performed an extensive literature review and convened several expert meetings. The expert group consisted of 12 neurologists with $\geq 10$ years of clinical experience in treating patients with $\mathrm{CH}$. The literature review and expert-group discussion resulted in seven items for screening $\mathrm{CH}$ being agreed upon, all of which were from the ICHD- $3 \beta$ criteria. ${ }^{17}$ The chosen items comprised two items for the duration of headache attack and unilateral pain, three items for the associated symptoms during a headache attack, and two items for the frequency of headache attacks and the duration of headache bout. We additionally included three items that are major characteristics of migraine (disability, nausea, and photophobia) to determine whether these items may be helpful in distinguishing between migraine and $\mathrm{CH}^{19}$ The item regarding severe pain from the ICHD-3 $\beta$ criteria was initially included, but it was subsequently discarded since disability was considered a better expression. Respondents were instructed to answer the items assessing duration, unilateral pain, and associated symptoms of the headache on the following 4-point Likert scale: $1=$ never, $2=$ rarely, $3=$ sometimes, and $4=$ frequently. A dichotomous scale (yes $=1$ and no $=0$ ) was used for items for the frequency of headache attacks, clustering features of headache attacks, and characteristics of migraine. The Likert scale was later transformed into a dichotomous scale for the analysis (never, rarely, or sometimes vs. frequently).

The questionnaire was then pilot tested with 10 patients at the headache clinics at 2 Korean hospitals (Dongtan Sacred Heart Hospital and Kangbuk Samsung Hospital). Patients were asked to complete the survey and participated in a brief interview thereafter, in which we asked whether the instructions and wording of the questionnaire were clear, and whether the questionnaire included the symptoms they were experiencing. This revealed that all of the questionnaire items were well understood, and no specific issues were raised. The resulting questionnaire comprising 10 items was administered to the study participants, who were first-visit patients presenting with headache.

In addition to administering the questionnaire, we obtained 
demographic and clinical information including age, sex, clinically diagnosed type of headache (gold standard), and duration of headache from hospital medical records. The gold-standard diagnosis used to test the validity of the questionnaire was the diagnosis made by a headache specialist based on the ICHD- $3 \beta$ after the patient had completed the questionnaire.

\section{Questionnaire validation and reliability testing}

We calculated Cronbach's alpha to check the internal consistency and reliability of the questionnaire. We used Cronbach's alpha $\geq 0.70$ as the standard for defining acceptable instrument reliability.

Considering the overlap between the characteristics of $\mathrm{CH}$ and other headaches (Supplementary Table 1 in the online-only Data Supplement), we decided to apply different weights to the scores for the various items. Items were scored 3 points if the difference in the prevalence of symptoms between $\mathrm{CH}$ and non- $\mathrm{CH}$ patients was $>60 \%, 2$ points if the difference was $50-60 \%$, and 1 point (i.e., no weighting) if the difference was $30-49.9 \%$.

The sensitivity and specificity values and the Youden Index (sensitivity+specificity-100) ${ }^{20}$ for various cutoff scores for the total scores of the final version of the questionnaire were then calculated. The corresponding Cronbach's alpha and the area under the curve (AUC) using the receiver operating characteristics test were also calculated.

The criterion validity was assessed by calculating the sensitivity and specificity for $\mathrm{CH}$. Furthermore, the positive likelihood ratio, negative likelihood ratio, PPV, and NPV were calculated. ${ }^{21}$ Likelihood ratios can range from 0 to infinity, where a value of 1 indicates no diagnostic value, values greater than 1 indicate a higher probability of disease (positive likelihood ratios), and values below 1 indicate a lower probability of disease (negative likelihood ratios). ${ }^{22}$

Considering that the questionnaire applies to people who have a headache, the PPV and NPV were additionally calculated using the estimated prevalence of $0.2 \%$ in the general population and $1 \%$ in headache clinics. ${ }^{4,23,24}$

\section{Statistical analysis}

Descriptive statistics were used for reporting the characteristics of participants and mean $\pm \mathrm{SD}$ values of each item. The independent-samples $t$-test was applied to continuous vari- ables, and the chi-square test was used for categorical variables. All statistical analyses were performed using the STATA software package (version 14, STATA Corporation, College Station, TX, USA), and two-sided probability values of $p<0.05$ were considered significant.

\section{RESULTS}

The participants were aged $42.9 \pm 11.5$ years, and $60.6 \%$ were female. Among the 304 participants, 12.4\% were diagnosed with $\mathrm{CH}$ (episodic in 41 and chronic in 1), 60.9\% were diagnosed with migraine (episodic in 141 and chronic in 66), 21.5\% were diagnosed with TTH (episodic in 53 and chronic in 20), and $5.3 \%$ were diagnosed with PSH. The mean ages of the patients with $\mathrm{CH}$, migraine, and other headaches (TTH or PSH) were $36.8,41.9$, and 48.1 years, respectively. Compared with non- $\mathrm{CH}$ patients, $\mathrm{CH}$ patients were more likely to be male $(p<0.01)$ (Table 1).

The differences in the responses to the questionnaire between $\mathrm{CH}$ and non- $\mathrm{CH}$ patients were $>60 \%$ for item 1 (headache improves within 3 hours), item 2 (headache is accompanied by conjunctival injection and/or tearing on the headache side), and item 4 (headache is accompanied by a sense of restlessness or agitation). These three items were scored 3 points if the patient responded positively. The difference in the prevalence was $50 \%$ to $60 \%$ for item 9 (headache is repeated intensively for over a week) and was scored 2 points. Other items with differences of 30-49.9\% were scored 1 point, and included items 3, 5, 6, and 10, and the male sex. Responses to item 7 (headache is accompanied by nausea or stomach sickness) and item 8 (photophobia) did not differ between patients with $\mathrm{CH}$ and migraine, and so these two items were discarded in the final scoring (Table 2). Consequently, the total score of the Cluster Headache Screening Questionnaire (CHSQ) ranged from 0 to 16 (Table 3 ). The CHSQ score was higher in $\mathrm{CH}$ patients than non- $\mathrm{CH}$ patients $(12.9 \pm 3.0$ vs. $3.4 \pm 2.5, p<$ 0.001 ) (Fig. 1). Cronbach's alpha was 0.74 , and the high accuracy of the scoring model was indicated by an AUC of 0.98 (Fig. 2).

The most-appropriate cutoff score as calculated based on the Youden Index was 8 points; $76.9 \%$ of the participants with a score of $>8$ points were found to have $\mathrm{CH}$. While $95.2 \%$ of $\mathrm{CH}$ patients scored $>8$ points, only $4 \%$ of non- $\mathrm{CH}$ patients

Table 1. Demographics and headache diagnoses of the patients $(n=340)$

\begin{tabular}{lccccc}
\hline & CH $(\boldsymbol{n}=\mathbf{4 2})$ & Migraine $(\boldsymbol{n}=\mathbf{2 0 7})$ & TH $(\boldsymbol{n}=\mathbf{7 3})$ & PSH $(\boldsymbol{n}=\mathbf{1 8})$ & $\boldsymbol{p}$ \\
\hline Age, years & $36.8 \pm 9.0$ & $41.9 \pm 11.3$ & $48.0 \pm 11.2$ & $48.2 \pm 11.0$ & $<0.001$ \\
Sex, male & $34(81.0)$ & $47(22.7)$ & $40(54.8)$ & $13(72.2)$ & $<0.001<$ \\
\hline
\end{tabular}

Data are $n(\%)$ or mean \pm SD values.

$\mathrm{CH}$ : cluster headache, PSH: primary stabbing headache, $\mathrm{TH}$ : tension-type headache. 
Table 2. Distribution of positive responses to each item of the CHSO according to the finally diagnosed headache types

\begin{tabular}{|c|c|c|c|c|}
\hline Item & $\begin{array}{c}\mathrm{CH} \\
(n=42)\end{array}$ & $\begin{array}{l}\text { Migraine } \\
(n=207)\end{array}$ & $\begin{array}{l}\text { TTH or PSH } \\
\quad(n=91)\end{array}$ & $\begin{array}{c}p^{*} \\
\text { (CH vs. Migraine) }\end{array}$ \\
\hline 1. Headache improves within 3 hours & $35(83.3)$ & $21(10.1)$ & $18(19.8)$ & $<0.001$ \\
\hline $\begin{array}{l}\text { 2. Headache is accompanied by conjunctival injection and/or tearing on the } \\
\text { headache side }\end{array}$ & $31(73.8)$ & $16(7.7)$ & $2(2.2)$ & $<0.001$ \\
\hline $\begin{array}{l}\text { 3. Headache is accompanied by nasal congestion and/or rhinorrhea on the } \\
\text { headache side }\end{array}$ & 16 (38.1) & 7 (3.4) & 0 & $<0.001$ \\
\hline 4. Headache is accompanied by sense of restlessness or agitation & $35(83.3)$ & $43(20.8)$ & $3(3.3)$ & $<0.001$ \\
\hline 5. Headache is unilateral on the right or left side & $38(90.5)$ & $104(50.2)$ & $26(28.6)$ & $<0.001$ \\
\hline 6. Headache limits you from doing what you need to do & $41(97.6)$ & $140(67.6)$ & $16(17.6)$ & $<0.001$ \\
\hline 7. Headache is accompanied by nausea and/or stomach sickness & $31(73.8)$ & $167(80.7)$ & $28(30.8)$ & 0.315 \\
\hline 8. Headache worsens when around light & $26(61.9)$ & $118(57.0)$ & $14(15.4)$ & 0.558 \\
\hline 9. Headache is repeated intensively for over a week & $37(88.1)$ & $59(28.5)$ & $28(30.8)$ & $<0.001$ \\
\hline 10. Headache recurs more than three times weekly & $36(85.7)$ & $96(46.4)$ & $49(53.9)$ & $<0.001$ \\
\hline
\end{tabular}

Data are $n(\%)$ values. Items answered on the Likert scale (items 1 to 5) were transformed into a dichotomous scale for the analysis (never, rarely, or sometimes vs. frequently). Detailed responses are presented in Supplementary Table 1 in the online-only Data Supplement.

${ }^{*} p$ values of all items among three groups and between $\mathrm{CH}$ and $\mathrm{TH}$ or $\mathrm{PSH}$ were $<0.001$.

$\mathrm{CH}$ : cluster headache, CHSO: Cluster Headache Screening Questionnaire, PSH: primary stabbing headache, TH: tension-type headache.

Table 3. Final 9-item Cluster Headache Screening Questionnaire scoring sheet

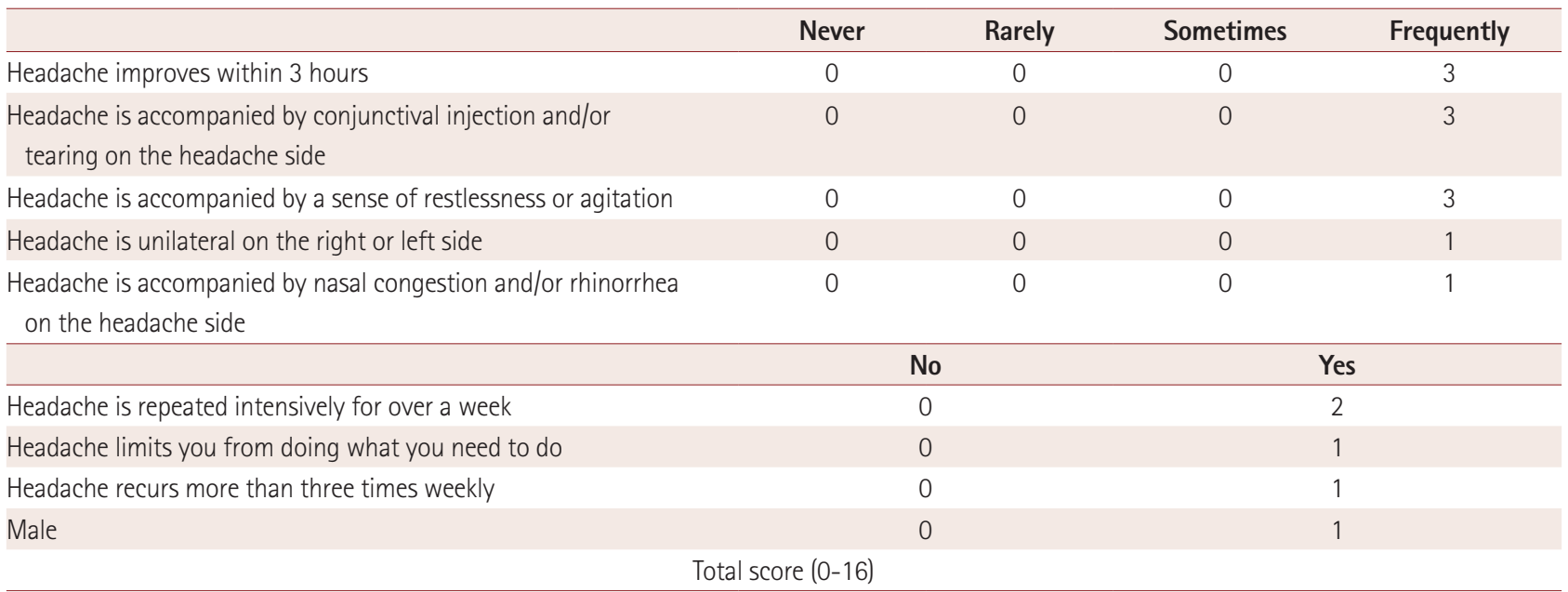

Sometimes represents less than half the time; frequently represents half the time or more.

achieved this. At the cutoff of $>8$ points, the sensitivity and specificity for $\mathrm{CH}$ were $95.2 \%$ and $96 \%$, respectively, while the PPV and NPV were $76.9 \%$ and $99.3 \%$, respectively. At a cutoff of $>10$ points, the risk score achieved sensitivity, specificity, PPV, and NPV values of $83.3 \%, 99.3 \%, 94.6 \%$, and $97.7 \%$, respectively (Table 4).

We estimated the PPV of the CHSQ tool when applying it to the prevalence of $\mathrm{CH}$ in the general population and headache clinics. Considering a presumed $1 \%$ prevalence of $\mathrm{CH}$ in headache clinics and $0.2 \%$ in the general population, the CHSQ achieved PPV and NPV values of $55.6 \%$ and $99.8 \%$ in headache clinics, and 32.6\% PPV and 100\% NPV in the general-population sample at a cutoff score of 10 points.

\section{DISCUSSION}

In this multicenter study, we developed a self-administered $\mathrm{CHQS}$ to rapidly identify individuals with $\mathrm{CH}$ from among various primary headache patients, based on the differences in the prevalence of symptoms between $\mathrm{CH}$ and non- $\mathrm{CH}$ patients. We found that the weighted scoring tool with nine questions for the male sex, attack duration, cranial autonomic symptoms, irritability, unilateral pain, disability, frequency, and clustering pattern is a valid and accurate tool for identifying $\mathrm{CH}$ among first-visit headache outpatients. When applied to first-visit headache patients, the sensitivity and specificity of the CHQS were $83.3 \%$ and $99.3 \%$, respectively, at a cutoff score of 10 points, and $95.2 \%$ and $96 \%$ at a cutoff score of 8 points, 


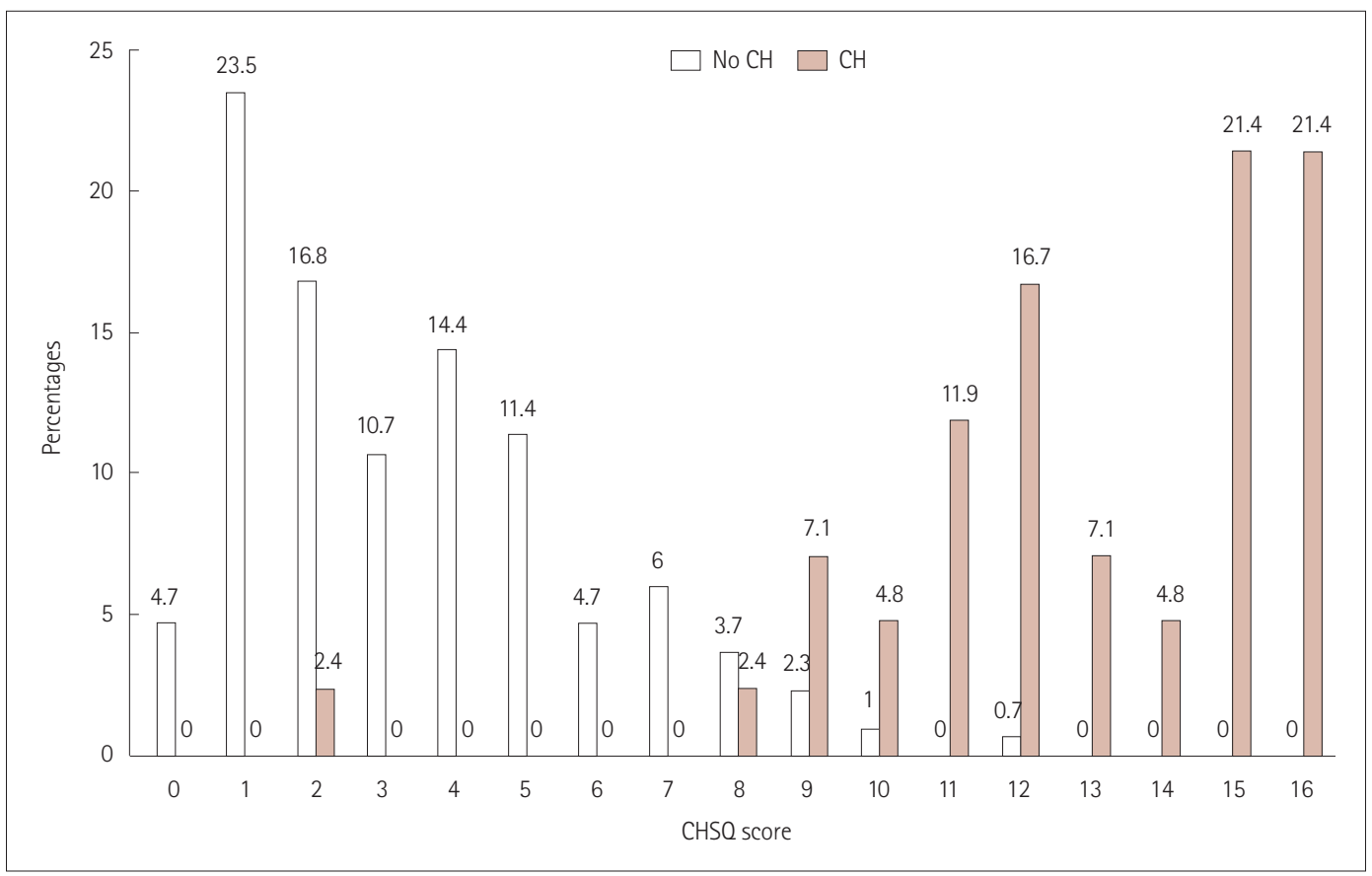

Fig. 1. Distribution of the $\mathrm{CHSO}$ scores between $\mathrm{CH}$ and non-CH patients. $\mathrm{CH}$ : cluster headache, $\mathrm{CHSO}$ : Cluster Headache Screening Questionnaire.

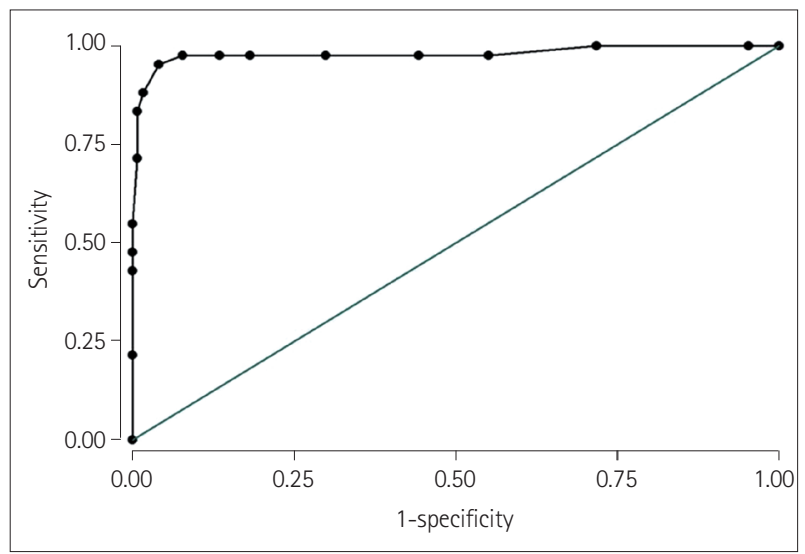

Fig. 2. ROC curve for the diagnostic accuracy of the Cluster Headache Screening Questionnaire scoring system. Area under ROC curve $=0.98$. ROC: receiver operating characteristic.

relative to a $\mathrm{CH}$ diagnosis performed using the ICHD-3 $\beta$.

$\mathrm{CH}$ is a severe primary headache disorder with characteristic clinical symptoms. Despite its well-defined clinical features and diagnostic criteria, the mean time to $\mathrm{CH}$ diagnosis is approximately 5 years; however, in one-third of cases this interval was found to be 12 years. ${ }^{8-10}$ Even when patients were referred to a headache center, $\geq 1$ year could elapse before a correct diagnosis. ${ }^{25}$ This indicates the need for a simple self-administered questionnaire tool for identifying $\mathrm{CH}$. Several groups have previously developed different screening questionnaires for diagnosing $\mathrm{CH}$. A three-item questionnaire comprising questions on unilaterality, attack duration, and ipsilateral conjunctival injection/lacrimation achieved a sensitivity
Table 4. Predictive parameters of the $\mathrm{CHSO}$ for detecting $\mathrm{CH}$

\begin{tabular}{ccc}
\hline Predictive parameter & CHSO score $>\mathbf{8}$ & CHSO score $>\mathbf{1 0}$ \\
\hline Sensitivity, \% & 95.2 & 83.3 \\
\hline Specificity, \% & 96.0 & 99.3 \\
PPV, \% & 76.9 & 94.6 \\
NPV, \% & 99.3 & 97.7 \\
PLR & 23.8 & 119.0 \\
NLR & 0.05 & 0.17 \\
PPV $^{*} \%$ & 4.6 & 32.6 \\
NPV $^{*}, \%$ & 100.0 & 100.0 \\
\hline
\end{tabular}

*Predicted PPV and NPV in the general population (estimated prevalence of $\mathrm{CH}$ in the general population of $0.2 \%$ ).

$\mathrm{CH}$ : cluster headache, CHSO: Cluster Headache Screening Questionnaire, NLR: negative likelihood ratio, NPV: negative predictive value, PLR: positive likelihood ratio, PPV: positive predictive value.

of $78 \%$ and a specificity of $100 \%$ in 96 patients with primary headache disorders, including 37 with $\mathrm{CH} .{ }^{14}$ Although this three-item questionnaire was reported to be very simple, some migraine patients with unilateral autonomic symptoms can be misclassified as $\mathrm{CH}$ with a yes/no question about autonomic symptoms. ${ }^{26,27}$ A 16-item self-administered questionnaire was validated in 71 patients with primary headache disorders, including 30 patients with $\mathrm{CH}$, which suggested an 8-question cluster was the best discriminatory tool, while applying 16 items would be too time-consuming. ${ }^{15}$ Furthermore, the Web-based Leiden University Cluster Headache Analysis program questionnaire was designed to diagnose $\mathrm{CH}$ among selfreported $\mathrm{CH}$ patients who fulfilled the inclusion screening questionnaire. ${ }^{13}$ Patients' awareness of $\mathrm{CH}$ symptoms may af- 
fect their responses to specific questionnaire items, and so we excluded previously diagnosed and self-reported $\mathrm{CH}$ patients from the present study.

While trigeminal autonomic symptoms are major features of $\mathrm{CH}$, mild infrequent cranial autonomic symptoms may be present during migraine attacks. ${ }^{28} \mathrm{~A}$ reliable screening tool should therefore have a high power for discriminating between $\mathrm{CH}$ and migraine. The present study showed that $32.7 \%$ of migraine patients reported lacrimation and/or injection during attacks (combining rare, occasional, and frequent responses). This result is similar to prevalence rates of $26.9-45 \%$ for autonomic symptoms in previous epidemiologic and clinic-based studies. ${ }^{27-29}$ However, regarding the frequency, only $7.6 \%$ of migraine patients reported frequent autonomic symptoms, compared with $73.8 \%$ of $\mathrm{CH}$ patients who reported frequent symptoms in the relevant item of the present questionnaire. Therefore, although autonomic symptoms are relatively prevalent in migraine patients, they could represent a powerful discriminating factor when using a Likert scale instead of a binary (yes/no) response option.

The responses to the questionnaire regarding migrainelike features also needed to be addressed, because disability, nausea, stomach sickness, and photophobia are frequently reported by patients with $\mathrm{CH},{ }^{9,30}$ Actually, disability was more common in patients with $\mathrm{CH}$ than in migraineurs (97.6\% vs. 67.6\%), and other features (nausea and photophobia) did not differ between the two groups. Therefore, as expected, the present study demonstrated that these migraine-like features might not be useful for differentiating $\mathrm{CH}$ from migraine.

$\mathrm{CH}$ is more prevalent in men than women. Therefore, male patients were scored 1 point in the CHSQ scoring tool. A previous report also suggested that the male sex might be valid screening option for $\mathrm{CH} .{ }^{13}$ Although a decreasing trend in the male/female ratio over time was suggested, the male-to-female ratio is still high, especially in Asian studies. ${ }^{624}$ Furthermore, considering the female predominance of migraine and other primary headache disorders, scoring for the male sex may contribute to the ability to discriminate between $\mathrm{CH}$ and migraine patients.

Epidemiologic studies have found clear discrepancies between $\mathrm{CH}$ diagnoses made by questionnaires and physicians. Among a total of 182 subjects diagnosed with suspected $\mathrm{CH}$ by the screening questionnaire, 4 cases $(2.2 \%)$ were confirmed as $\mathrm{CH}$ and the remaining were finally diagnosed as migraine with trigeminal autonomic symptoms in a German population study. ${ }^{16}$ Other population-based studies have also found low PPVs (3.1-12.6\%) for screening questionnaires in diagnosing $\mathrm{CH}^{31,32} \mathrm{~A}$ screening method with higher validity is therefore required in epidemiologic studies of $\mathrm{CH}$. When applied to the prevalence of $\mathrm{CH}$ in the general population $(0.2 \%)$, the estimated PPV and NPV were $32.6 \%$ and $100 \%$, respectively, for a cutoff of 10 points. These values suggest that it might be useful to apply the CHSQ in epidemiologic studies.

The merit of the CHSQ tool is that the cutoff score for diagnosing $\mathrm{CH}$ is flexible and can be adjusted depending on the characteristics of the population (e.g., specialized headache clinic, primary care clinic, or general population-based study). Although we speculated an optimal cutoff score of 8 points for diagnosing $\mathrm{CH}$ in headache clinics, the PPV of the tool can be improved by using a cutoff score of 10 points. Conversely, researchers using this tool in population-based studies might choose a lower cutoff score in order to increase the sensitivity for screening purposes. Another merit of the CHSQ is its short length. The CHSQ is composed of eight items (plus a score for the male sex) and takes $<5 \mathrm{~min}$ to complete, and so it is expected to be easily applicable in generalpopulation studies and achieve high response rates. Unlike previous studies, this study included patients with PSH, which are frequently encountered in headache clinics. ${ }^{33}$

The present study was subject to several limitations. First, although this screening questionnaire was tested in first-visit headache patients before a diagnosis was performed, all participants were enrolled in the neurology department of a secondary or tertiary hospital, which may have resulted in selection bias of the enrolled patients. Therefore, the CHSQ should be further validated in a primary-care setting with a larger population. Second, we enrolled first-visit headache patients aged 18-65 years among the Korean population and only one patient with chronic $\mathrm{CH}$ was included. This tool might therefore not be generalizable to screening chronic $\mathrm{CH}$ and so should be validated in other language. Finally, the gold standard of this study was a clinical diagnosis based upon the criteria in the previous ICHD- $3 \beta$ version. The main changes in the current ICHD-3 criteria were deleting recently added associated symptoms (fullness in the ear, forehead and facial flushing) and extending the maximum remission period of chronic $\mathrm{CH}$ up to 3 months. Although the presence of ear fullness and facial flushing have been reported to not influence the diagnosis of $\mathrm{CH}$, the CHSQ should still be validated with the current ICHD-3 criteria. ${ }^{34}$

In conclusion, we have developed a highly reliable and simple eight-item questionnaire plus a score for the male sex that can reliably identify patients with $\mathrm{CH}$ among first-visit headache patients. The application of the CHSQ tool will facilitate the early identification of $\mathrm{CH}$ in clinics. Further validation is warranted in other clinical settings and different populations.

\section{Supplementary Materials}

The online-only Data Supplement is available with this article at https://doi.org/10.3988/jcn.2019.15.1.90. 


\section{Conflicts of Interest}

Dr. Cho was involved as a site investigator of multicenter trial sponsored by Otsuka Korea, Eli Lilly and Company, Korea BMS, and Eisai Korea, and worked as an advisory member for Teva, and received research support from Hallym University Research Fund 2016 and received Academic award of Myung In Pharm.Ltd, and received lecture honoraria from Yuyu Pharmaceutical Company. All other authors have no financial conflicts of interest.

\section{Acknowledgements}

This study was supported by a grant from Korean Neurological Association (gran number KNA-16-MI-09).

\section{REFERENCES}

1. Goadsby PJ. Pathophysiology of cluster headache: a trigeminal autonomic cephalgia. Lancet Neurol 2002;1:251-257.

2. Jensen RM, Lyngberg A, Jensen RH. Burden of cluster headache. Cephalalgia 2007;27:535-541.

3. Abu Bakar N, Torkamani M, Tanprawate S, Lambru G, Matharu M, Jahanshahi $\mathrm{M}$. The development and validation of the Cluster Headache Quality of life scale (CHQ). J Headache Pain 2016;17:79.

4. Fischera M, Marziniak M, Gralow I, Evers S. The incidence and prevalence of cluster headache: a meta-analysis of population-based studies. Cephalalgia 2008;28:614-618.

5. Ekbom K, Svensson DA, Träff H, Waldenlind E. Age at onset and sex ratio in cluster headache: observations over three decades. Cephalalgia 2002;22:94-100.

6. Moon HS, Park JW, Lee KS, Chung CS, Kim BK, Kim JM, et al. Clinical features of cluster headache patients in Korea. J Korean Med Sci 2017;32:502-506.

7. Gaul C, Finken J, Biermann J, Mostardt S, Diener HC, Müller O, et al. Treatment costs and indirect costs of cluster headache: a health economics analysis. Cephalalgia 2011;31:1664-1672.

8. Klapper JA, Klapper A, Voss T. The misdiagnosis of cluster headache: a nonclinic, population-based, internet survey. Headache 2000;40:730735.

9. van Vliet JA, Eekers PJ, Haan J, Ferrari MD; Dutch RUSSH Study Group. Features involved in the diagnostic delay of cluster headache. J Neurol Neurosurg Psychiatry 2003;74:1123-1125.

10. Viana M, Tassorelli C, Allena M, Nappi G, Sjaastad O, Antonaci F. Diagnostic and therapeutic errors in trigeminal autonomic cephalalgias and hemicrania continua: a systematic review. J Headache Pain 2013;14:14

11. Robbins MS, Starling AJ, Pringsheim TM, Becker WJ, Schwedt TJ. Treatment of cluster headache: the American Headache Society evidence-based guidelines. Headache 2016;56:1093-1106.

12. Leroux E, Valade D, Taifas I, Vicaut E, Chagnon M, Roos C, et al. Suboccipital steroid injections for transitional treatment of patients with more than two cluster headache attacks per day: a randomised, double-blind, placebo-controlled trial. Lancet Neurol 2011;10:891-897.

13. Wilbrink LA, Weller CM, Cheung C, Stijnen T, Haan J, Ferrari MD, et al. Stepwise web-based questionnaires for diagnosing cluster headache: LUCA and QATCH. Cephalalgia 2013;33:924-931.

14. Dousset V, Laporte A, Legoff M, Traineau MH, Dartigues JF, Brochet B. Validation of a brief self-administered questionnaire for cluster headache screening in a tertiary center. Headache 2009;49:64-70.
15. Torelli P, Beghi E, Manzoni GC. Validation of a questionnaire for the detection of cluster headache. Headache 2005;45:644-652.

16. Katsarava Z, Obermann M, Yoon MS, Dommes P, Kuznetsova J, Weimar C, et al. Prevalence of cluster headache in a population-based sample in Germany. Cephalalgia 2007;27:1014-1019.

17. Headache Classification Committee of the International Headache Society (IHS). The International Classification of Headache Disorders, 3rd edition (beta version). Cephalalgia 2013;33:629-808.

18. Preacher KJ, MacCallum RC. Exploratory factor analysis in behavior genetics research: factor recovery with small sample sizes. Behav Genet 2002;32:153-161.

19. Lipton RB, Dodick D, Sadovsky R, Kolodner K, Endicott J, Hettiarachchi J, et al. A self-administered screener for migraine in primary care: the ID Migraine validation study. Neurology 2003;61:375-382.

20. Youden WJ. Index for rating diagnostic tests. Cancer 1950;3:32-35.

21. Lalkhen AG, McCluskey A. Clinical tests: sensitivity and specificity. Contin Educ Anaesth Crit Care Pain 2008;8:221-223.

22. McGee S. Simplifying likelihood ratios. J Gen Intern Med 2002;17: 646-649.

23. Kim BK, Chu MK, Lee TG, Kim JM, Chung CS, Lee KS. Prevalence and impact of migraine and tension-type headache in Korea. J Clin Neurol 2012;8:204-211.

24. Lin KH, Wang PJ, Fuh JL, Lu SR, Chung CT, Tsou HK, et al. Cluster headache in the Taiwanese-a clinic-based study. Cephalalgia 2004; 24:631-638.

25. Voiticovschi-Iosob C, Allena M, De Cillis I, Nappi G, Sjaastad O, Antonaci F. Diagnostic and therapeutic errors in cluster headache: a hospital-based study. J Headache Pain 2014;15:56.

26. Barbanti P, Fabbrini G, Pesare M, Vanacore N, Cerbo R. Unilateral cranial autonomic symptoms in migraine. Cephalalgia 2002;22:256-259.

27. Barbanti P, Aurilia C, Dall'Armi V, Egeo G, Fofi L, Bonassi S. The phenotype of migraine with unilateral cranial autonomic symptoms documents increased peripheral and central trigeminal sensitization. A case series of 757 patients. Cephalalgia 2016;36:1334-1340.

28. Lai TH, Fuh JL, Wang SJ. Cranial autonomic symptoms in migraine: characteristics and comparison with cluster headache. J Neurol Neurosurg Psychiatry 2009;80:1116-1119.

29. Obermann M, Yoon MS, Dommes P, Kuznetsova J, Maschke M, Weimar C, et al. Prevalence of trigeminal autonomic symptoms in migraine: a population-based study. Cephalalgia 2007;27:504-509.

30. Taga A, Russo M, Manzoni GC, Torelli P. Cluster headache with accompanying migraine-like features: a possible clinical phenotype. Headache 2017;57:290-297.

31. Torelli P, Beghi E, Manzoni GC. Cluster headache prevalence in the Italian general population. Neurology 2005;64:469-474.

32. Katsarava Z, Dzagnidze A, Kukava M, Mirvelashvili E, Djibuti M, Janelidze M, et al. Prevalence of cluster headache in the Republic of Georgia: results of a population-based study and methodological considerations. Cephalalgia 2009;29:949-952.

33. Kim BK, Cho SJ, Kim BS, Sohn JH, Kim SK, Cha MJ, et al. Comprehensive application of the International Classification of Headache Disorders third edition, beta version. J Korean Med Sci 2016;31:106113.

34. de Coo IF, Wilbrink LA, Haan J, Ferrari MD, Terwindt GM. Evaluation of the new ICHD-III beta cluster headache criteria. Cephalalgia 2016;36:547-551. 